\title{
550 - Caregiver Burden and Geriatric Patients Undergoing Electroconvulsive Therapy in the COVID-19 Era
}

Valeriya Tsygankova, Maria A. Grullon, Bobbi Woolwine, Ashley Subler, Parker Schwab, Adriana Patricia Hermida, MD

\section{Introduction}

Caregivers are essential in providing valuable patient information to medical providers. With limited available research investigating caregiver burden among those caring for patients receiving ECT and the new life challenges presented by the COVID-19 pandemic, it is more important now than ever to take a closer look at the caregivers' experiences.

\section{Methods}

Emory's Wesley Woods Geriatric Hospital is one of the busiest ECT services in the country with around 2000 treatments completed every year. In this chart review, researchers are looking at caregivers' responses to the Zarit Caregiver Burden Interview. This assessment includes 22 questions assessing the caregivers' current emotional state in order to examine the impact of the patient's condition on

caregiver wellbeing. The score ranges from 0 (no burden) to 88 (severe burden). Other objective assessments completed with ECT patients are BDI (Beck Depression Inventory), BDI Suicide, Sheehan Disability Scale, ECCA (Electrocompulsive Cognitive Assessment), GAD-7 (Generalized Anxiety Disorder Scale), and CGI (Clinical Global Impression). The purpose of this chart review is to investigate whether the higher severity of depression in patients correlates with higher levels of caregiver burden and whether the pandemic contributed to caregiver burden.

\section{Results}

The Zarit Caregiver Burden Interview has been administered on twelve caregivers ( $n=12 ; 6$ male and 6 female), all providing care for patients currently undergoing ECT for depressive symptoms. Out of the twelve caregivers, $66.6 \%$ reported little caregiver burden, $16.6 \%$ reported mild to moderate burden, and $16.6 \%$ reported moderate to severe burden. Additional patients are being interviewed in the ECT suite and further data collected will be presented at the 2021 IPA Virtual International Congress.

\section{Conclusion}

As the world adjusts to life in the COVID-19 era, caregivers face new challenges in providing care for vulnerable, at-risk populations. Caregivers of patients receiving ECT facilitate frequent COVID testing and limited visitation policies while continuing to provide care and emotional support to patients with advanced age and a mental illness. Administrating the Zarit Caregiver Burden Interview can help providers look at the degree of burden in caregivers of patients receiving ECT for depressive symptoms and provide better caregiving strategies during and post pandemic.

\section{1 - Case Report: De Archambault's syndrome in the early stage of dementia with Lewy bodies}

Takashi Suehiro $^{1,2}$, Yuto Satake ${ }^{2}$, Mamoru Hashimoto ${ }^{2,3}$, Hisahiro $\mathrm{Yu}^{1}$, Manabu Ikeda $^{2}$

1. Hanwa Izumi Hospital

2. Department of Psychiatry, Osaka University Graduate School of Medicine

3. Department of Neuropsychiatry, Kindai University Faculty of Medicine

Background: Dementia with Lewy bodies (DLB) is the second most common form of degenerative dementia after Alzheimer's disease. In some patients with DLB, relatively rare delusions are known to emerge, such as Othello syndrome, delusional parasitosis and delusion of duplication. Erotomania, also known as de Clerambault's syndrome, is characterized by the delusion that a person has fallen in love with the patient. It occasionally appears secondary to psychiatric disorders and organic brain diseases. However, there have been no reports on cases secondary to patients with DLB. 
Case presentation: The patient was an 83-year-old woman who lived alone. Mild cognitive impairment appeared at the age of 82 years. Soon after, she had the delusional conviction that her family doctor was in love with her. Her symptoms, such as gradually progressive cognitive impairment, cognitive fluctuations, and parkinsonism, indicated DLB. Although small doses of quetiapine, brexpiprazole and risperidone were prescribed for the treatment of the delusion, each of them was discontinued soon because of the adverse reactions. Finally, the delusion was successfully treated with a small dose of blonanserin without sever side effects.

Discussions and Conclusions: This case report suggests the possibility of de Clerambault's syndrome during the early stages of DLB. Recently, psychiatric-onset DLB has increasingly gained attention in recent years. Further accumulation of knowledge about delusions in patients with DLB for an early diagnosis.

\title{
552 - Humanitude Care on Patients with Dementia and Delirium in Acute Hospital Improves Outcomes
}

\author{
Thuy-Anh Giang, Cheng Ling Jie, Chua Min Jia, Philip Yap
}

Background/ Objectives: Older patients with dementia and/or delirium often have challenging behaviours such as refusal of care and aggression. These cause much distress to both healthcare staff and patients, increase burden of care and put older patients at risk of functional decline. Humanitude, a methodology of care developed by Gineste and Marescotti, is a relationship- centred and compassionate care approach that aims to enable patients. The aim of this study is to investigate the effectiveness of Humanitude on older patients' well-being, mobility and activities of daily living (ADLs).

Design: Quasi-experimental, non-equivalent controlled trial design.

Setting: Acute care hospital

Participants: Twenty patients diagnosed with dementia and/ or delirium were recruited from two geriatric wards. Ten were in the Humanitude ward and the other ten in a conventional ward received usual care for older patients served as concurrent controls.

Intervention: Patients in Humanitude ward received Humanitude care by trained healthcare workers during day-to-day care, which is based on the 4 pillars of gaze, speech, touch and verticality (maintaining an upright position). Every patient encounter utilizing Humanitude techniques follow a structured care sequence that helps draw the patient into the care relationship.

Measurements: The outcome measures include Modified Perme ICU Mobility Score, Bradford Wellbeing Profile and Modified Barthel Index (MBI).

Results: There was significant improvement of median score within the Humanitude group from admission to discharge in mobility (admission: 9.0 [0-27] vs discharge 19.5 [1-36], $p=0.002^{* *}$ ), MBI (admission: 20 [0.0-46] vs discharge: 54.3 [3-81], $\mathrm{p}=0.002^{* *}$ ) and well-being (admission: 7.0 [1-15] vs discharge 20.0 [8-26], $\left.p=0.002^{* *}\right)$. The median increase in the score of Humanitude group was also significantly higher than usual care group in mobility (Humanitude: 8 [1-24] vs usual care 0 [-9-16], $p=$ $0.02 *$ ), MBI (Humanitude: 17.5 [3-64] vs usual care $0[-3-17], p=<0.001 * * *$ ), and well-being (Humanitude: 11 [6-20] vs usual care $0[-5-4], p=<0.001 * * *$ ).

Conclusion: Humanitude care improves outcomes in mobility, ADL function and well-being for patients with dementia and/ or delirium in the acute hospital.

(Word count: 329) 